\title{
STRINGY HODGE NUMBERS AND VIRASORO ALGEBRA
}

\author{
VICTOR V. BATYREV
}

\begin{abstract}
In this paper we define for singular varieties $X$ a rational number $c_{\mathrm{st}}^{1, n-1}(X)$ which is a stringy version of the product of Chern numbers $c_{1}$ and $c_{n-1}$ We show that the number $c_{\mathrm{st}}^{1, n-1}(X)$ can be expressed via stringy Hodge numbers of singular $X$ in the same way as $c_{1} c_{n-1}$ expresses via usual Hodge numbers for smooth manifolds. Our result provides some evidences for the existence of quantum cohomology theory of singular varieties $X$ based on representation of the Virasoro algebra whose central charge is the rational number $e_{\text {st }}(X)$ which equals the stringy Euler number of $X$.
\end{abstract}

\section{Introduction}

Let $X$ be an arbitrary smooth $n$-dimensional projective variety. It was discovered by Libgober and Wood that the product of the Chern classes $c_{1}(X) c_{n-1}(X)$ depends only on the Hodge numbers of $X$ [11]. This result has been used by Eguchi, Jinzenji and Xiong in their approach to the quantum cohomology of $X$ via a representation of the Virasoro algebra with the central charge $c_{n}(X)[8,9]$.

We recall that the $E$-polynomial of $X$ is defined as

$$
E(X ; u, v):=\sum_{p, q}(-1)^{p+q} h^{p, q}(X) u^{p} v^{q},
$$

where $h^{p, q}(X)=\operatorname{dim} H^{q}\left(X, \Omega_{X}^{p}\right)$ are Hodge numbers of $X$. Using the Hirzebruch-Riemann-Roch theorem, Libgober and Wood [11] have proved the following equality (see also results of Borisov [6] and Salamon [12]):

Theorem 1.1.

$$
\left.\frac{d^{2}}{d u^{2}} E_{\mathrm{st}}(X ; u, 1)\right|_{u=1}=\frac{3 n^{2}-5 n}{12} c_{n}(X)+\frac{c_{1}(X) c_{n-1}(X)}{6} .
$$

By Poincaré duality for $X$, one immediately obtains the following equivalent reformulation of the above equality:

Theorem 1.2. Let $X$ be an arbitrary smooth $n$-dimensional projective variety. Then $c_{1}(X) c_{n-1}(X)$ can be expressed via the Hodge numbers of $X$ using the following equality

$$
\sum_{p, q}(-1)^{p+q} h^{p, q}(X)\left(p-\frac{n}{2}\right)^{2}=\frac{n}{12} c_{n}(X)+\frac{1}{6} c_{1}(X) c_{n-1}(X),
$$

Received December 19, 1999. 
where

$$
c_{n}(X)=\sum_{p, q}(-1)^{p+q} h^{p, q}(X)
$$

is the Euler number of $X$.

In particular, one has:

Corollary 1.3. Let $X$ be an arbitrary smooth $n$-dimensional projective variety with $c_{1}(X)=0$. Then the Hodge numbers of $X$ satisfy the following equation

$$
\sum_{p, q}(-1)^{p+q} h^{p, q}(X)\left(p-\frac{n}{2}\right)^{2}=\frac{n}{12} \sum_{p, q}(-1)^{p+q} h^{p, q}(X) .
$$

Remark 1.4. We note that if $X$ is a $K 3$-surface, then the relation 1.3 is equivalent to the equality $c_{2}(X)=24$. For smooth Calabi-Yau 4-folds $X$ the equality 1.3 has been observed by Sethi, Vafa, and Witten [13]. It is equivalent to the equality

$$
c_{4}(X)=6\left(8-h^{1,1}(X)+h^{2,1}(X)-h^{3,1}(X)\right),
$$

if $h^{1,0}(X)=h^{2,0}(X)=h^{3,0}(X)=0$.

There are a lot of examples of Calabi-Yau varieties $X$ having at worst Gorenstein canonical singularities which are hypersurfaces and complete intersections in Gorenstein toric Fano varieties [1,3]. It has been shown in [5] that for all these singular Calabi-Yau varieties $X$ one can define so called stringy Hodge numbers $h_{\mathrm{st}}^{p, q}(X)[2]$. Moreover, the stringy Hodge numbers of Calabi-Yau complete intersections in Gorenstein toric varieties agree with the topological mirror duality test [4]. It is natural to expect that one has the same kind of identity for stringy Hodge numbers of singular Calabi-Yau varieties as for usual Hodge numbers of smooth Calabi-Yau manifolds, i.e.,

(1) $\sum_{p, q}(-1)^{p+q} h_{\mathrm{st}}^{p, q}(X)\left(p-\frac{n}{2}\right)^{2}=\frac{n}{12} \sum_{p, q}(-1)^{p+q} h_{\mathrm{st}}^{p, q}(X)=\frac{n}{12} e_{\mathrm{st}}(X)$.

This paper is to show that the formula (1) holds true. Moreover, one can define a rational number $c_{\mathrm{st}}^{1, n-1}(X)$, a stringy version $c_{1}(X) c_{n-1}(X)$, such that the stringy analogue of the equation in 1.2

$$
\sum_{p, q}(-1)^{p+q} h_{\mathrm{st}}^{p, q}(X)\left(p-\frac{n}{2}\right)^{2}=\frac{n}{12} e_{\mathrm{st}}(X)+\frac{1}{6} c_{\mathrm{st}}^{1, n-1}(X),
$$

holds true provided the stringy Hodge numbers of $X$ exist. 


\section{Stringy Hodge numbers}

Recall our general approach to the notion of stringy Hodge numbers $h_{\mathrm{st}}^{p, q}(X)$ for projective algebraic varieties $X$ with canonical singularites (see [2]). Our main definition in [2] can be reformulated as follows:

Definition 2.1. Let $X$ be an arbitrary $n$-dimensional projective variety with at worst log-terminal singularities, $\rho: Y \rightarrow X$ a resolution of singularities whose exceptional locus $D$ is a divisor with normally crossing components $D_{1}, \ldots, D_{r}$. We set $I:=\{1, \ldots, r\}$ and $D_{J}:=\bigcap_{j \in J} D_{j}$ for all $J \subseteq I$, where $D_{J}=Y$ if $J=\emptyset$. Define the stringy $E$-function of $X$ to be

$$
E_{\mathrm{st}}(X ; u, v):=\sum_{\emptyset \subseteq J \subseteq I} E\left(D_{J} ; u, v\right) \prod_{j \in J}\left(\frac{u v-1}{(u v)^{a_{j}+1}-1}-1\right),
$$

where the rational numbers $a_{1}, \ldots, a_{r}$ are determined by the equality

$$
K_{Y}=\rho^{*} K_{X}+\sum_{i=1}^{r} a_{i} D_{i} .
$$

Then the stringy Euler number of $X$ is defined as

$$
e_{\mathrm{st}}(X):=\lim _{u, v \rightarrow 1} E_{\mathrm{st}}(X ; u, v)=\sum_{\emptyset \subseteq J \subseteq I} c_{n-|J|}\left(D_{J}\right) \prod_{j \in J}\left(\frac{-a_{j}}{a_{j}+1}\right),
$$

where $c_{n-|J|}\left(D_{J}\right)$ is the Euler number of $D_{J}$ (we set $c_{n-|J|}\left(D_{J}\right)=0$ if $D_{J}$ is empty).

Remark 2.2. It is important that the above definitions do not depend on the choice of a desingularization $\rho: Y \rightarrow X[2]$.

Definition 2.3. Let $X$ be an arbitrary $n$-dimensional projective variety with at worst Gorenstein canonical singularites. We say that stringy Hodge numbers of $X$ exist, if $E_{\mathrm{st}}(X ; u, v)$ is a polynomial, i.e.,

$$
E_{\mathrm{st}}(X ; u, v)=\sum_{p, q} a_{p, q}(X) u^{p} v^{q} .
$$

Under the assumption that $E_{\mathrm{st}}(X ; u, v)$ is a polynomial, we define the stringy Hodge numbers $h_{\mathrm{st}}^{p, q}(X)$ to be $(-1)^{p+q} a_{p, q}(X)$.

Remark 2.4. In the above definitions, the condition that $X$ has at worst logterminal singularities means that $a_{i}>-1$ for all $i \in I$; the condition that $X$ has at worst Gorenstein canonical singularities is equivalent for $a_{i}$ to be nonnegative integers for all $i \in I$ (see [10]).

The following statement has been proved in [2]:

Theorem 2.5. Let $X$ be an arbitrary $n$-dimensional projective variety with at worst Gorenstein canonical singularites. Assume that stringy Hodge numbers of $X$ exist. Then they have the following properties:

(i) $h_{\mathrm{st}}^{0,0}(X)=h_{\mathrm{st}}^{n, n}(X)=1$; 
(ii) $h_{\mathrm{st}}^{p, q}(X)=h_{\mathrm{st}}^{n-p, n-q}(X)$ and $h_{\mathrm{st}}^{p, q}(X)=h_{\mathrm{st}}^{q, p}(X) \forall p, q$;

(iii) $h_{\mathrm{st}}^{p, q}(X)=0 \forall p, q>n$.

\section{The number $c_{\mathrm{st}}^{1, n-1}(X)$}

Definition 3.1. Let $X$ be an arbitrary $n$-dimensional projective variety $X$ having at worst log-terminal singularities and $\rho: Y \rightarrow X$ is a desingularization with normally crossing irreducible components $D_{1}, \ldots, D_{r}$ of the exceptional locus. We define the number

$$
c_{\mathrm{st}}^{1, n-1}(X):=\sum_{\emptyset \subseteq J \subseteq I} \rho^{*} c_{1}(X) c_{n-|J|-1}\left(D_{J}\right) \prod_{j \in J}\left(\frac{-a_{j}}{a_{j}+1}\right),
$$

where $\rho^{*} c_{1}(X) c_{n-|J|-1}\left(D_{J}\right)$ is considered as the intersection number of the 1cycle $c_{n-|J|-1}\left(D_{J}\right) \in A_{1}\left(D_{J}\right)$ with the $\rho$-pullback of the class of the anticanonical $\mathbb{Q}$-divisor of $X$.

Remark 3.2. It is not clear a priori that the number $c_{\mathrm{st}}^{1, n-1}(X)$ in the above the definition does not depend on the choice of a desingularization $\rho$. Later we shall show this independence.

The proof of the next obvious statement is left to the reader:

Proposition 3.3. For any smooth $n$-dimensional projective variety $V$, one has

$$
\left.\frac{d}{d u} E(V ; u, 1)\right|_{u=1}=\frac{n}{2} c_{n}(V) .
$$

Proposition 3.4. For any $n$-dimensional projective variety $X$ having at worst log-terminal singularities, one has

$$
\left.\frac{d}{d u} E_{\mathrm{st}}(X ; u, 1)\right|_{u=1}=\frac{n}{2} e_{\mathrm{st}}(X) .
$$

Proof. By definition 2.1, we have

$$
E_{\mathrm{st}}(X ; u, 1)=\sum_{\emptyset \subseteq J \subseteq I} E\left(D_{J} ; u, 1\right) \prod_{j \in J}\left(\frac{u-1}{u^{a_{j}+1}-1}-1\right) .
$$

Applying 3.3 to every smooth submanifold $D_{J} \subset Y$, we obtain

$$
\begin{aligned}
\left.\frac{d}{d u} E_{\mathrm{st}}(X ; u, 1)\right|_{u=1}= & \sum_{\emptyset \subseteq J \subseteq I} \frac{(n-|J|)}{2} c_{n-|J|}\left(D_{J}\right) \prod_{j \in J}\left(\frac{-a_{j}}{a_{j}+1}\right)+ \\
& +\sum_{\emptyset \subseteq J \subseteq I} \frac{|J|}{2} c_{n-|J|}\left(D_{J}\right) \prod_{j \in J}\left(\frac{-a_{j}}{\left(a_{j}+1\right)}\right) \\
= & \frac{n}{2} \sum_{\emptyset \subseteq J \subseteq I} c_{n-|J|}\left(D_{J}\right) \prod_{j \in J}\left(\frac{-a_{j}}{a_{j}+1}\right)=\frac{n}{2} e_{\mathrm{st}}(X) .
\end{aligned}
$$


Proposition 3.5. Let $V$ be a smooth projective algebraic variety of dimension $n$ and $W \subset V$ a smooth irreducible divisor on $V$ or empty divisor (the latter means that $\left.\mathcal{O}_{V}(W) \cong \mathcal{O}_{V}\right)$. Then

$$
c_{1}\left(\mathcal{O}_{V}(W)\right) c_{n-1}(V)=c_{n-1}(W)+c_{1}\left(\mathcal{O}_{W}(W)\right) c_{n-2}(W)
$$

where $c_{n-1}(W)$ is considered to be zero if $W=\emptyset$.

Proof. Consider the short exact sequnce

$$
\left.0 \rightarrow T_{W} \rightarrow T_{V}\right|_{W} \rightarrow \mathcal{O}_{W}(W) \rightarrow 0,
$$

where $T_{W}$ and $T_{V}$ are tangent sheaves on $W$ and $V$. It gives the following the relation between Chern polynomials

$$
\begin{array}{r}
\left(1+c_{1}\left(\mathcal{O}_{W}(W)\right) t\right)\left(1+c_{1}(W) t+c_{2}(W) t^{2}+\cdots+c_{n-1}(W) t^{n-1}\right)= \\
1+c_{1}\left(\left.T_{V}\right|_{W}\right) t+c_{2}\left(\left.T_{V}\right|_{W}\right) t^{2}+c_{n-1}\left(\left.T_{V}\right|_{W}\right) t^{n-1} .
\end{array}
$$

Comparing the coefficients by $t^{n-1}$ and using $c_{n-1}\left(\left.T_{V}\right|_{W}\right)=c_{1}\left(\mathcal{O}_{V}(W)\right) c_{n-1}(V)$, we come to the required equality.

Corollary 3.6. Let $Y$ be a smooth projective variety, $D_{1}, \ldots, D_{r}$ smooth irreducible divisors with normal crossings, $I:=\{1, \ldots, r\}$. Then for all $J \subseteq I$ and for all $j \in J$ one has

$$
c_{1}\left(\mathcal{O}_{D_{J \backslash\{j\}}}\left(D_{j}\right)\right) c_{n-|J|}\left(D_{J \backslash\{j\}}\right)-c_{n-|J|}\left(D_{J}\right)=c_{1}\left(\mathcal{O}_{D_{J}}\left(D_{j}\right)\right) c_{n-|J|-1}\left(D_{J}\right),
$$

where $D_{J}$ is the complete intersection $\bigcap_{j \in J} D_{j}$.

Proof. One sets in $3.5 V:=D_{J \backslash\{j\}}$ and $W:=D_{J}$.

Proposition 3.7. Let $\rho: Y \rightarrow X$ be a desingularization as in 3.1. Then

$$
\begin{aligned}
& \sum_{\emptyset \subseteq J \subseteq I} c_{1}\left(D_{J}\right) c_{n-|J|-1}\left(D_{J}\right) \prod_{j \in J}\left(\frac{-a_{j}}{a_{j}+1}\right)= \\
& c_{\mathrm{st}}^{1, n-1}(X)+\sum_{\emptyset \subseteq J \subseteq I}\left(\sum_{j \in J}\left(a_{j}+1\right) c_{n-|J|}\left(D_{J}\right)\right) \prod_{j \in J}\left(\frac{-a_{j}}{a_{j}+1}\right) .
\end{aligned}
$$

Proof. Using the formula

$$
c_{1}(Y)=\rho^{*} c_{1}(X)+\sum_{i \in I}-a_{i} c_{1}\left(\mathcal{O}_{Y}\left(D_{i}\right)\right)
$$

and the adjunction formula for every complete intersection $D_{J}(J \subseteq I)$, we obtain

$$
c_{1}\left(D_{J}\right)=\left.\rho^{*} c_{1}(X)\right|_{D_{J}}+\sum_{j \in J}\left(-a_{j}-1\right) c_{1}\left(\mathcal{O}_{D_{J}}\left(D_{j}\right)\right)+\sum_{j \in I \backslash J}\left(-a_{j}\right) c_{1}\left(\mathcal{O}_{D_{J}}\left(D_{j}\right)\right) .
$$


Therefore

$$
\begin{aligned}
\sum_{\emptyset \subseteq J \subseteq I} c_{1}\left(D_{J}\right) c_{n-|J|-1}\left(D_{J}\right) \prod_{j \in J}\left(\frac{-a_{j}}{a_{j}+1}\right)=c_{\mathrm{st}}^{1, n-1}(X)+ \\
\sum_{\emptyset \subseteq J \subseteq I}\left(\sum_{j \in J}\left(-a_{j}-1\right) c_{1}\left(\mathcal{O}_{D_{J}}\left(D_{j}\right)\right) c_{n-|J|-1}\left(D_{J}\right)\right) \prod_{j \in J}\left(\frac{-a_{j}}{a_{j}+1}\right)+ \\
\sum_{\emptyset \subseteq J \subseteq I}\left(\sum_{j \in I \backslash J}\left(-a_{j}\right) c_{1}\left(\mathcal{O}_{D_{J}}\left(D_{j}\right)\right) c_{n-|J|-1}\left(D_{J}\right)\right) \prod_{j \in J}\left(\frac{-a_{j}}{a_{j}+1}\right) .
\end{aligned}
$$

Using 3.6, we obtain

$$
\begin{aligned}
& \sum_{j \in J}\left(-a_{j}-1\right) c_{1}\left(\mathcal{O}_{D_{J}}\left(D_{j}\right)\right) c_{n-|J|-1}\left(D_{J}\right)= \\
& \sum_{j \in J}\left(-a_{j}-1\right)\left(c_{1}\left(\mathcal{O}_{D_{J \backslash\{j\}}}\left(D_{j}\right)\right) c_{n-|J|}\left(D_{J \backslash\{j\}}\right)-c_{n-|J|}\left(D_{J}\right)\right) .
\end{aligned}
$$

By substitution (4) to (3), we come to the required equality, because

$$
\begin{aligned}
\sum_{\emptyset \subseteq J \subseteq I}( & \left.\sum_{j \in J}\left(-a_{j}-1\right) c_{1}\left(\mathcal{O}_{D_{J \backslash\{j\}}}\left(D_{j}\right)\right) c_{n-|J|}\left(D_{J \backslash\{j\}}\right)\right) \prod_{j \in J}\left(\frac{-a_{j}}{a_{j}+1}\right)+ \\
& \sum_{\emptyset \subseteq J \subseteq I}\left(\sum_{j \in I \backslash J}\left(-a_{j}\right) c_{1}\left(\mathcal{O}_{D_{J}}\left(D_{j}\right)\right) c_{n-|J|-1}\left(D_{J}\right)\right) \prod_{j \in J}\left(\frac{-a_{j}}{a_{j}+1}\right)=0 .
\end{aligned}
$$

Theorem 3.8. Let $X$ be an arbitrary $n$-dimensional projective variety variety with at worst log-terminal singularities. Then

$$
\left.\frac{d^{2}}{d u^{2}} E_{\mathrm{st}}(X ; u, 1)\right|_{u=1}=\frac{3 n^{2}-5 n}{12} e_{\mathrm{st}}(X)+\frac{1}{6} c_{\mathrm{st}}^{1, n}(X) .
$$

Proof. Using the equalities

$$
\frac{d}{d u}\left(\frac{u-1}{u^{a+1}-1}-1\right)_{u=1}=\frac{-a}{2(a+1)}, \frac{d^{2}}{d u^{2}}\left(\frac{u-1}{u^{a+1}-1}-1\right)_{u=1}=\frac{a(a+2)}{6(a+1)},
$$

together with the identities in 1.1 and 3.3 for every submanifold $D_{J} \subset Y$, we obtain 


$$
\begin{aligned}
&\left.\frac{d^{2}}{d u^{2}} E_{\mathrm{st}}(X ; u, 1)\right|_{u=1}=\sum_{\emptyset \subseteq J \subseteq I} \frac{c_{1}\left(D_{J}\right) c_{n-|J|-1}\left(D_{J}\right)}{6} \prod_{j \in J}\left(\frac{-a_{j}}{a_{j}+1}\right)+ \\
& c_{n-|J|}(D J) \frac{3(n-|J|)^{2}-5(n-|J|)}{12} \prod_{j \in J}\left(\frac{-a_{j}}{a_{j}+1}\right)+ \\
& \sum_{\emptyset \subseteq J \subseteq I} \frac{(n-|J|)|J| c_{n-|J|}\left(D_{J}\right)}{2} \prod_{j \in J}\left(\frac{-a_{j}}{a_{j}+1}\right)+ \\
& \sum_{\emptyset \subseteq J \subseteq I} \frac{c_{n-|J|}\left(D_{J}\right)(|J|-1)|J|}{4} \prod_{j \in J}\left(\frac{-a_{j}}{a_{j}+1}\right)+ \\
& \sum_{\emptyset \subseteq J \subseteq I} \frac{c_{n-|J|}\left(D_{J}\right)\left(-\sum_{j \in J}\left(a_{j}+2\right)\right)}{6} \prod_{j \in J}\left(\frac{-a_{j}}{a_{j}+1}\right) .
\end{aligned}
$$

By 3.7, the first term of the above equals

$$
\frac{1}{6} c_{\mathrm{st}}^{1, n-1}(X)+\frac{1}{6} \sum_{\emptyset \subseteq J \subseteq I}\left(\sum_{j \in J}\left(a_{j}+1\right) c_{n-|J|}\left(D_{J}\right)\right) \prod_{j \in J}\left(\frac{-a_{j}}{a_{j}+1}\right) .
$$

Now the required statement follows from the equality

$$
\begin{aligned}
\frac{\sum_{j \in J}\left(a_{j}+1\right)}{6}+\frac{3(n-|J|)^{2}-5(n-|J|)}{12}+\frac{(n-|J|)|J|}{2}+ \\
\frac{(|J|-1)|J|}{4}+\frac{-\sum_{j \in J}\left(a_{j}+2\right)}{6}=\frac{3 n^{2}-5 n}{12} .
\end{aligned}
$$

Corollary 3.9. The number $c_{\mathrm{st}}^{1, n}(X)$ does not depend on the choice of the desingularization $\rho: Y \rightarrow X$.

Proof. By 3.4 and $3.8, c_{\mathrm{st}}^{1, n}(X)$ can be computed in terms of derivatives of the stringy $E$-function of $X$. But the stringy $E$-function does not depend on the choice of a desingularization [2].

Corollary 3.10. Let $X$ be a projective variety with at worst Gorenstein canonical singularities. Assume that the stringy Hodge numbers of $X$ exist. Then

$$
\sum_{p, q}(-1)^{p+q} h_{\mathrm{st}}^{p, q}(X)\left(p-\frac{n}{2}\right)^{2}=\frac{n}{12} e_{\mathrm{st}}(X)+\frac{1}{6} c_{\mathrm{st}}^{1, n-1}(X) .
$$

Proof. The equality follows immediately from 3.8 using the properties of the stringy Hodge numbers 2.5. 
Corollary 3.11. Assume that the canonical class of $X$ is numerically trivial. Then $c_{\mathrm{st}}^{1, n-1}(X)=0$. In particular, for Calabi-Yau varieties with at worst Gorenstein canonical singularities we have

$$
\left.\frac{d^{2}}{d u^{2}} E_{\mathrm{st}}(X ; u, 1)\right|_{u=1}=\frac{3 n^{2}-5 n}{12} e_{\mathrm{st}}(X),
$$

and therefore stringy Hodge numbers of X satisfy the identity (1) provided that stringy numbers exist.

Example 3.12. Let $\Delta$ be an $n$-dimensional reflexive polyhedron and $\Delta^{*}$ its polar polyhedron [1]. We denote by $\mathbb{P}_{\Delta}$ the Gorenstein toric Fano variety associated with $\Delta$. If $\Theta$ a convex lattice polyhedron of dimension $k$ then we denote by $v(\Theta)$ the integer $k ! \operatorname{vol}_{k}(\Theta)$ where $\operatorname{vol}_{k}(\Theta)$ is the $k$-dimensional volume of $\Theta$ with respect to the lattice. It is known that $e_{\mathrm{st}}\left(\mathbb{P}_{\Delta}\right)=v\left(\Delta^{*}\right)$ (see Cor. 7.7 [5]). One can ask about the meaning of the new invariant $c_{\mathrm{st}}^{1, n}$ in terms of $\Delta$. Assume that $\mathbb{P}_{\Delta}$ has a smooth crepant toric desingularization $\rho: \widehat{\mathbb{P}_{\Delta}} \rightarrow \mathbb{P}_{\Delta}$ which is defined by a triangulation $\mathcal{T}$ of the boundary $\partial \Delta^{*}$ by regular simplices. By 3.1 we obtain that

$$
c_{\mathrm{st}}^{1, n}\left(\mathbb{P}_{\Delta}\right)=c_{1}\left(\widehat{\mathbb{P}_{\Delta}}\right) c_{n-1}\left(\widehat{\mathbb{P}_{\Delta}}\right) .
$$

Now we use that fact that in the nonsigular case the Chern class $c_{n-1}\left(\widehat{\mathbb{P}_{\Delta}}\right)$ is the sum of all 1-dimensional strata in $\widehat{\mathbb{P}_{\Delta}}[7]$. These 1-dimensional strata correspond to $(d-2)$-dimensional simplices in the triangulation $\mathcal{T}$ of $\partial \Delta^{*}$. Let $\tau$ be such a $(d-2)$-dimensional simplex which is a common boundary of two $(d-1)$ dimensional ones $\sigma_{1}$ and $\sigma_{2}$. Denote by $Z_{\tau}$ the 1 -cycle on $\widehat{\mathbb{P}_{\Delta}}$ corresponding to $\tau$. It follows from the description of $c_{1}$ in terms of a canonical piecewise linear function having value 1 on $\partial \Delta^{*}$ that the intersection number of $Z_{\tau}$ with $c_{1}\left(\widehat{\mathbb{P}_{\Delta}}\right)$ is zero unless $\sigma_{1}$ and $\sigma_{2}$ belong to different faces $\gamma_{1}^{*}$ and $\gamma_{2}^{*}$ of codimension 1 of $\Delta^{*}$. The faces $\gamma_{1}^{*}$ and $\gamma_{2}^{*}$ are dual to two vertices $\gamma_{1}, \gamma_{2} \in \Delta$ and the intersection number $c_{1}\left(\widehat{\mathbb{P}_{\Delta}}\right) Z_{\tau}$ equals just the integral length $v\left(\left[\gamma_{1}, \gamma_{2}\right]\right)$ of the segment $\left[\gamma_{1}, \gamma_{2}\right]$. Let $\left[\gamma_{1}, \gamma_{2}\right]^{*} \subset \Delta^{*}$ be the dual to $\left[\gamma_{1}, \gamma_{2}\right](d-2)$-dimensional face. Then $\left[\gamma_{1}, \gamma_{2}\right]^{*}$ contains exactly $v\left(\left[\gamma_{1}, \gamma_{2}\right]^{*}\right)$ simplices from the triangulation $\mathcal{T}$, because for all such simplices one has $v(\tau)=1$. The above arguments show that the number

$$
c_{\mathrm{st}}^{1, n}\left(\mathbb{P}_{\Delta}\right)=c_{1}\left(\widehat{\mathbb{P}_{\Delta}}\right) \sum_{\tau \in \mathcal{T}} Z_{\tau}
$$

equals

$$
\sum_{\left[\gamma, \gamma^{\prime}\right] \subset \Delta} v\left(\left[\gamma, \gamma^{\prime}\right]\right) \cdot v\left(\left[\gamma, \gamma^{\prime}\right]^{*}\right)
$$

So we have

$$
c_{\mathrm{st}}^{1, n-1}\left(\mathbb{P}_{\Delta}\right)=\sum_{\theta \subset \Delta, \operatorname{dim} \theta=1} v(\theta) \cdot v\left(\theta^{*}\right)
$$




\section{Virasoro algebra}

Recall that the Virasoro algebra with the central charge $c$ consists of operators $L_{n}(m \in \mathbb{Z})$ satisfying the relations

$$
\left[L_{n}, L_{m}\right]=(n-m) L_{n+m}+c \frac{n^{3}-n}{12} \delta_{n+m, 0} \quad n, m \in \mathbb{Z} .
$$

For arbitrary compact Kähler manifold $X$, Eguchi et. al have proposed in [8, 9] a new approach to its quantum cohomology and to its Gromov-Witten invariants for all genera $g$ using so called the Virasoro condition:

$$
L_{n} Z=0, \forall n \geq-1,
$$

where

$$
Z=\exp F=\exp \left(\sum_{g \geq 0} \lambda^{2 g-2} F_{g}\right)
$$

is the partition function of the topological $\sigma$-model with the target space $X$ and $F_{g}$ the free energy function corresponding to the genus $g$. In this approach, the central charge $c$ acts as the multiplication by $c_{n}(X)$. Moreover, all Virasoro operators $L_{n}$ can be explicitly written in terms of elements of a basis of the cohomology of $X$, their gravitational descendants and the action of $c_{1}(X)$ on the cohomology by the multiplication. In particular the commutator relation

$$
\left[L_{1}, L_{-1}\right]=2 L_{0}
$$

implies precisely the identity of Libgober and Wood in the form

$$
\begin{aligned}
\sum_{p, q}(-1)^{p+q} h^{p, q}(X)\left(\frac{n+1}{2}-p\right)(p- & \left.\frac{n-1}{2}\right)= \\
& \frac{1}{6}\left(\frac{3-n}{2} c_{n}(X)-c_{1}(X) c_{n-1}(X)\right) .
\end{aligned}
$$

Now let $X$ be a projective algebraic variety with at worst log-terminal singularities. We conjecture that there exists an analogous approach to the quantum cohomology as well as to the Gromov-Witten invariants of $X$ for all genera using the Virasoro algebra in such a way that for any resolution of singularities $\rho: Y \rightarrow X$ the corresponding Virasoro operators can be explicitly computed via the numbers $a_{i}$ appearing in the formula

$$
K_{X}=\rho^{*} K_{X}+\sum_{i=1}^{r} a_{i} D_{i}
$$

and bases in cohomology of all complete intersections $D_{J}$ together with the multiplicative actions of $c_{1}\left(D_{J}\right)$ in them. We consider our main result 3.8 as an evidence in favor of this conjecture. 


\section{References}

[1] V.V. Batyrev, Dual polyhedra and mirror symmetry for Calabi-Yau hypersurfaces in toric varieties, J. Algebraic Geom., 3 (1994), 493-535.

[2] _ Stringy Hodge numbers of varieties with Gorenstein canonical singularities, Integrable systems and algebraic geometry (Kobe/Kyoto, 1997), World Sci. Publishing, River Edge, NJ, 1998, pp. 1-31.

[3] V.V. Batyrev and L.A. Borisov, Dual cones and mirror symmetry for generalized CalabiYau manifolds, Mirror Symmetry II, AMS/IP Stud. Adv. Math., 1, Amer. Math. Soc., Providence, RI, 1997 pp. 65-80.

[4] _ Mirror Duality and String-Theoretic Hodge Numbers, Invent. Math., 126 (1996), 183-203.

[5] V.V. Batyrev and D. Dais, Strong McKay correspondence, string-theoretic Hodge numbers and mirror symmetry, Topology, 35 (1996), 901-929.

[6] L.A. Borisov, On the Betti numbers and Chern classes of varieties with trivial odd cohomology groups, preprint 1997, alg-geom/9703023

[7] V.I. Danilov, Geometry of toric varieties, Uspekhi Mat. Nauk 33 (1978), 97-154

[8] T. Eguchi, K. Hori, and Ch.-Sh. Xiong, Quantum cohomology and Virasoro algebra, Phys. Lett. B 402 (1997), 71-80.

[9] T. Eguchi, M. Jinzenji, and Ch.-Sh. Xiong, Quantum cohomology and free field representation, Nucl. Phys. B 510 (1998), 608-622.

[10] Y. Kawamata, K. Matsuda, and K. Matsuki, Introduction to the minimal model problem, Algebraic geometry, Sendai, 1985, Adv. Stud. Pure Math., 10, North-Holland, Amsterdam-New York, 1987, pp. 283-360.

[11] A.S. Libgober and J.W. Wood, Uniqueness of the complex structure on Kahler manifolds of certain homotopy types, J. Diff. Geom. 32 (1990), 139-154.

[12] S.M. Salamon, On the cohomology of Kahler and hyper-Kahler manifolds, Topology 35 (1996), 137-155.

[13] S. Sethi, C. Vafa, and E. Witten, Constraints on low-dimensional string compactifications, Nucl. Phys. B 480 (1996), 213-224.

Mathematisches Institut, Universität Tübingen, Auf der Morgenstelle 10, 72076 TÜBINGEN, GERMANY

E-mail address: batyrev@bastau.mathematik.uni-tuebingen.de 